\title{
Lorentzian para-Sasakian Manifolds Admitting a New Type of Quarter-symmetric Non-metric $\xi$-connection
}

\author{
Sudhakar K Chaubey* and Uday Chand De \\ (Communicated by Cihan Özgür )
}

\begin{abstract}
We define a new type of quarter-symmetric non-metric $\xi$-connection on an $L P$-Sasakian manifold and prove its existence. We provide its application in the general theory of relativity. To validate the existence of the quarter-symmetric non-metric $\xi$-connection on an $L P$-Sasakian manifold, we give a non-trivial example in dimension 4 and verify our results.
\end{abstract}

Keywords: Lorentzian para-Sasakian manifold; quarter-symmetric non-metric connection; conformal curvature tensors; Einstein space; Einstein's field equations.

AMS Subject Classification (2010): Primary: 53D10; Secondary: 53C25; 53D15.

\section{Introduction}

The semi-Riemannian geometry attracts the researchers because of its capabilities to resolve the many issues of science, technology and medical, and their allied areas. A differentiable manifold $M$ of dimension $n$ equipped with a semi-Riemannian metric $g$, whose signature is $(p, q)(p+q=n)$, known as an $n$-dimensional semi-Riemannian manifold. In particular, if we take $p=1, q=n-1$ or $p=n-1, q=1$, then the semiRiemannian manifold $M$ converts into the well known Lorentzian manifold. To start the study of Lorentzian manifold $M$, the causal character of the vectors played a significant role and hence it becomes the convenient choice for the researchers to study the general theory of relativity and cosmology. Space-time is the stage of present modeling of the physical world: a torsionless, time oriented Lorentzian manifold. In describing the gravity of the space-time, the Riemannian curvature $R$, the Ricci tensor $S$ and the scalar curvature $r$ played a crucial role. The observation, in cosmology, showed that the matter of space-time is isotropic and homogeneous. In [8], De et al. proved that, under certain condition, the matter of the space-time is filled with the isotropic and homogeneous fluid. Many researchers studied the properties of the space-time. We cite ([8], [14], [16], [30]) and their references.

A linear connection $\tilde{\nabla}$ on a semi-Riemannian manifold $M$ is said to be symmetric if the torsion tensor $\tilde{T}$ of $\tilde{\nabla}$ vanishes identically, otherwise it is non-symmetric. In particular, if $\tilde{T}(X, Y)=\eta(Y) X-\eta(X) Y, \forall X, Y \in$ $\chi(M)$, then it is known as a semi-symmetric connection [12], where $\eta$ is a 1-form associated with the semiRiemannian metric $g$ as $\eta(\cdot)=g(\cdot, \xi), \xi$ is a vector field of type $(1,0)$ and $\chi(M)$ denotes the set of differentiable vector fields of $M$. Also, if

$$
\tilde{T}(X, Y)=\eta(Y) \phi X-\eta(X) \phi Y, \quad \forall X, Y \in \chi(M),
$$

then the linear connection $\tilde{\nabla}$ is said to be a quarter-symmetric connection [13]. A semi-symmetric (quartersymmetric) connection $\tilde{\nabla}$ is said to be metric if $\tilde{\nabla} g=0$, otherwise it is non-metric, that is, $\tilde{\nabla} g \neq 0$ [15]. Since then, the properties of the quarter-symmetric metric (non-metric) connections on different structures have been studied by many geometers. For more details, we refer ([3], [5], [9], [21], [23], [25], [27]) and the references there in. 
The study of the symmetric spaces played a major role in the development of the differential geometry [4]. A semi-Riemannian manifold $M$ of dimension $n$ is said to be semi-symmetric if the non-vanishing curvature tensor $R$ of $M$ satisfies the curvature condition $R \cdot R=0$ [28]. A Ricci semi-symmetric manifold $M$ with dimension $n$ is an $n$-dimensional semi-Riemannian manifold provided the non-zero Ricci tensor $S$ of $M$ satisfies the condition $R \cdot S=0$. It is well known that a semi-symmetric manifold is Ricci semi-symmetric but converse part is not true, in general. Many geometers studied the properties of the semi-symmetric and Ricci semi-symmetric manifolds. For instance, we see [11], [24].

The above fruitful information motivate us to define a new type of the quarter-symmetric non-metric $\xi$ connection on an LP-Sasakian manifold. We organize our work as: After introduction in Section 1, we brief some basic results of the LP-Sasakian manifold in Section 2. Section 3 concerns with the existence of a new type of quarter-symmetric non-metric $\xi$-connection. In Section 4, we establish the relation between the curvature tensors of $\tilde{\nabla}$ and $\nabla$, and some basic results of the curvature tensors are given. Section 5 deals with the applications of the Lorentzian para-Sasakian manifolds equipped with a quarter-symmetric non-metric $\xi$-connection in the general theory of relativity. We ensure the existence of the quarter-symmetric non-metric $\xi$-connection on an LP-Sasakian manifold by providing a non-trivial example in Section 6 . We also verify our results.

\section{Lorentzian para-Sasakian manifolds}

Motivated by the Sasakian structure, K. Matsumoto [17] defined the notion of the Lorentzian para-Sasakian manifolds in 1989. Mihai et al. [19] developed the same concept independently and proved many interesting results. Since then, many researchers studied the geometrical and physical properties of the Lorentzian paraSasakian manifolds. For instance, see [2], [7], [20] and [22].

A quartet $(\phi, \xi, \eta, g)$ defined on an $n$-dimensional smooth manifold $M$, where $\phi$ is a tensor field of type $(1,1)$, $\xi$, a unit timelike vector field, $\eta$, a 1-form and $g$ is the Lorentzian metric satisfying

$$
\begin{gathered}
\phi^{2} X=X+\eta(X) \xi, \quad \eta(\xi)=-1, \\
\eta(\phi X)=0, \quad \phi \xi=0, \quad g(X, \xi)=\eta(X), \quad g(\phi X, \phi Y)=g(X, Y)+\eta(X) \eta(Y)
\end{gathered}
$$

for all $X, Y \in \chi(M)$, is known as a Lorentzian para-contact metric structure and the manifold $M$ associated with the metric $g$ is called the Lorentzian para-contact metric manifold $[17,18]$.

If moreover,

$$
\left(\nabla_{X} \phi\right)(Y)=[g(X, Y)+\eta(X) \eta(Y)] \xi+[X+\eta(X) \xi] \eta(Y)
$$

and

$$
\nabla_{X} \xi=\phi X \Longleftrightarrow\left(\nabla_{X} \eta\right)(Y)=g(\phi X, Y)
$$

hold on $M$ for all $X, Y \in \chi(M)$, then the Lorentzian para-contact metric manifold $M$ is known as a Lorentzian para-Sasakian (briefly, LP-Sasakian) manifold $[17,18]$. Here $\nabla$ is the Levi-Civita connection of $M$. It is noticed that the $n$-dimensional $L P$-Sasakian manifold $M$ satisfies the following relations.

$$
\begin{gathered}
R(X, Y) \xi=\eta(Y) X-\eta(X) Y, \\
R(\xi, X) Y=g(X, Y) \xi-\eta(Y) X \\
S(X, \xi)=(n-1) \eta(X), \\
\eta(R(X, Y) Z)=\eta(X) g(Y, Z)-\eta(Y) g(X, Z)
\end{gathered}
$$

foll all $X, Y, Z \in \chi(M)$, where $R$ and $S$ denote the curvature tensor and the Ricci tensor of $M$, respectively.

An $L P$-Sasakian manifold $M$ of dimension $n$ is said to be Einstein if

$$
S=a g,
$$

where $a$ is a constant. 


\section{Existence of the quarter-symmetric non-metric $\xi$-connection on a Lorentzian para-Sasakian manifold}

Let $M$ be an $n$-dimensional $L P$-Sasakian manifold. Then a linear connection $\tilde{\nabla}$ defined on $M$ as

$$
\tilde{\nabla}_{X} Y=\nabla_{X} Y+\eta(Y) \phi X-g(\phi X, \phi Y) \xi, \quad \forall X, Y \in \chi(M)
$$

is known as a quarter-symmetric non-metric connection if it satisfies the equations (1.1) and

$$
\left(\tilde{\nabla}_{X} g\right)(Y, Z)=\eta(Y)\{g(\phi X, \phi Z)-g(\phi X, Z)\}+\eta(Z)\{g(\phi X, \phi Y)-g(\phi X, Y)\} .
$$

If in addition, $\tilde{\nabla}$ satisfies $\tilde{\nabla} \xi=0$, then $\tilde{\nabla}$ is said to be a quarter-symmetric non-metric $\xi$-connection on $M$. Recently, in [6], authors studied the properties of semi-symmetric metric $P$-connection on a Riemannian manifold.

Next, we have to prove the existence of the quarter-symmetric non-metric $\xi$-connection on an $n$-dimensional $L P$-Sasakian manifold in the following theorem.

Theorem 3.1. There exists a unique quarter-symmetric non-metric $\xi$-connection $\tilde{\nabla}$ defined by (3.1) on an $n$-dimensional LP-Sasakian manifold $M$.

Proof. Suppose the linear connection $\tilde{\nabla}$ defined on an $n$-dimensional $L P$-Sasakian manifold $M$ is connected with $\nabla$ by the relation

$$
\tilde{\nabla}_{X} Y=\nabla_{X} Y+H(X, Y)
$$

for all $X, Y \in \chi(M)$, where $H$ denotes a tensor field of type $(1,2)$. By definition of torsion tensor, the equation (3.3) leads to

$$
\tilde{T}(X, Y)=H(X, Y)-H(Y, X), \quad \forall X, Y \in \chi(M)
$$

We have

$$
g(H(X, Y), Z)-g(H(Y, X), Z)=\eta(Y) g(\phi X, Z)-\eta(X) g(\phi Y, Z) .
$$

The equation (3.3) leads

$$
\begin{aligned}
\left(\tilde{\nabla}_{X} g\right)(Y, Z) & =g\left(Y, \nabla_{X} Z-\tilde{\nabla}_{X} Z\right)+g\left(Z, \nabla_{X} Y-\tilde{\nabla}_{X} Y\right) \\
& =-H^{\prime}(X, Y, Z)
\end{aligned}
$$

where

$$
H^{\prime}(X, Y, Z)=g(H(X, Y), Z)+g(Y, H(X, Z))
$$

From the equations (3.4) and (3.6), we have

$$
\begin{aligned}
& g(\tilde{T}(X, Y), Z)+g(\tilde{T}(Z, X), Y)+g(\tilde{T}(Z, Y), X) \\
& \quad=2 g(H(X, Y), Z)-H^{\prime}(X, Y, Z)+H^{\prime}(Z, X, Y)-H^{\prime}(Y, X, Z),
\end{aligned}
$$

which takes the following form

$$
\begin{aligned}
2 g(H(X, Y), Z)= & g(\tilde{T}(X, Y), Z)+g\left(\tilde{T}^{\prime}(X, Y), Z\right)+g\left(\tilde{T}^{\prime}(Y, X), Z\right) \\
& -\left(\tilde{\nabla}{ }_{X} g\right)(Y, Z)+\left(\tilde{\nabla}_{Z} g\right)(X, Y)-\left(\tilde{\nabla}_{Y} g\right)(X, Z) \\
= & g(\tilde{T}(X, Y), Z)+g\left(\tilde{T}^{\prime}(X, Y), Z\right)+g\left(\tilde{T}^{\prime}(Y, X), Z\right) \\
& -2 \eta(Z)\{g(\phi X, \phi Y)-g(\phi X, Y)\},
\end{aligned}
$$

where the equations (3.2) and (3.6) are used. Here $\tilde{T}^{\prime}$ is defined as

$$
g\left(\tilde{T}^{\prime}(X, Y), Z\right)=g(\tilde{T}(Z, X), Y)=\eta(X) g(\phi Z, Y)-\eta(Z) g(\phi X, Y) .
$$

In consequence of the equations (3.8) and (3.9), we get

$$
H(X, Y)=\eta(Y) \phi X-g(\phi X, \phi Y) \xi
$$

In view of the equations (3.3) and (3.10), we can easily get the equation (3.1). From the equation (3.1), it is obvious that $\tilde{\nabla} \xi=0$. Hence the linear connection $\tilde{\nabla}$ defined on an $n$-dimensional $L P$-Sasakian manifold is a quarter-symmetric non-metric $\xi$-connection. The converse part of the Theorem 3.1 is obvious. 
The covariant derivative of $\eta(Y)=g(Y, \xi)$ with respect to $\tilde{\nabla}$ along the vector field $X$, follows by the equations (3.1) and (3.2), gives

$$
\begin{aligned}
\left(\tilde{\nabla}_{X} \eta\right)(Y) & =\left(\tilde{\nabla}_{X} g\right)(Y, \xi) \\
& =\left(\nabla_{X} \eta\right)(Y)-g(\phi X, \phi Y) .
\end{aligned}
$$

From the equation (3.11), we can write

$$
\left(\tilde{\nabla}_{X} \eta\right)(Y)-\left(\tilde{\nabla}_{Y} \eta\right)(X)=\left(\nabla_{X} \eta\right)(Y)-\left(\nabla_{Y} \eta\right)(X) .
$$

This shows that the 1-form $\eta$ is closed with respect to $\nabla$ if and only if it is also closed with respect to $\tilde{\nabla}$. Thus we can state:

Proposition 3.1. Let an n-dimensional LP-Sasakian manifold $M$ admits a quarter-symmetric non-metric $\xi$-connection $\tilde{\nabla}$. Then the 1-form $\eta$ is closed with respect to $\nabla$ if and only if it is also closed with respect to $\tilde{\nabla}$.

Replacing $Y$ by $\phi Y$ in (3.1), we find that

$$
\tilde{\nabla}_{X}(\phi Y)=\nabla_{X}(\phi Y)+\eta(\phi Y) \phi X-g\left(\phi X, \phi^{2}(Y)\right) \xi
$$

which follows from the equations (2.1) and (2.2)

$$
\left(\tilde{\nabla}_{X} \phi\right)(Y)=\left(\nabla_{X} \phi\right)(Y)-\eta(Y) X-\eta(Y) \eta(X) \xi-g(\phi X, Y) \xi .
$$

\section{Curvature tensor with respect to the quarter-symmetric non-metric $\xi$-connection}

With the help of the equations (2.1)-(2.4) and (3.1), we have

$$
\begin{aligned}
\tilde{\nabla}_{X} \tilde{\nabla}_{Y} Z= & \nabla_{X}\left\{\nabla_{Y} Z+\eta(Z) \phi Y-g(\phi Y, \phi Z) \xi\right\} \\
& +\eta\left(\nabla_{Y} Z+\eta(Z) \phi Y-g(\phi Y, \phi Z) \xi\right) \phi X \\
& -g\left(\phi \nabla_{Y} Z+\eta(Z) \phi^{2} Y-g(\phi Y, \phi Z) \phi \xi, \phi X\right) \xi \\
= & \nabla_{X} \nabla_{Y} Z+g(\phi X, Z) \phi Y+\eta\left(\nabla_{X} Z\right) \phi Y+\eta(Z) g(X, Y) \xi \\
& +2 \eta(Z) \eta(Y) \eta(X) \xi+\eta(Z) \eta(Y) X+\eta(Z) \phi \nabla_{X} Y \\
& -g(X, \phi Z) \eta(Y) \xi-g\left(\phi \nabla_{X} Y, \phi Z\right) \xi-\eta(Z) g(\phi Y, X) \xi \\
& -g\left(\phi Y, \phi \nabla_{X} Z\right) \xi-g(\phi Y, \phi Z) \phi X+\eta\left(\nabla_{Y} Z\right) \phi X+g(Y, Z) \phi X \\
& +\eta(Y) \eta(Z) \phi X-g\left(\phi \nabla_{Y} Z, \phi X\right) \xi-\eta(Z) g(Y, \phi X) \xi .
\end{aligned}
$$

Let $\tilde{R}$ denotes the curvature tensor with respect to the quarter-symmetric non-metric $\xi$-connection, defined as

$$
\tilde{R}(X, Y) Z=\tilde{\nabla}_{X} \tilde{\nabla}_{Y} Z-\tilde{\nabla}_{Y} \tilde{\nabla}_{X} Z-\tilde{\nabla}_{[X, Y]} Z
$$

for all $X, Y, Z \in \chi(M)$. Interchanging $X$ and $Y$ in the equation (4.1) and then using it along with the equations (3.1) and (4.1) in the equation (4.2), we obtain

$$
\begin{gathered}
\tilde{R}(X, Y) Z=R(X, Y) Z+g(\phi X, Z) \phi Y+\eta(Z) \eta(Y) X-g(X, \phi Z) \eta(Y) \xi \\
-g(\phi Y, Z) \phi X-\eta(Z) \eta(X) Y+g(Y, \phi Z) \eta(X) \xi .
\end{gathered}
$$

Considering a frame field and then contracting the equation (4.3) along the vector field $X$, we find

$$
\tilde{S}(Y, Z)=S(Y, Z)+g(Y, Z)+n \eta(Y) \eta(Z)-(1+\psi) g(Y, \phi Z),
$$

which is equivalent to

$$
\tilde{Q} Y=Q Y+Y+n \eta(Y) \xi-(1+\psi) \phi Y,
$$

where $\psi$ is the trace of $\phi$. Taking a frame field and contracting the equation (4.5) along the vector field $Y$ we lead

$$
\tilde{r}=r-\psi(\psi+1) \text {. }
$$

Now we prove the basic properties of the curvature tensor $\tilde{R}$ in the following theorem. 
Theorem 4.1. Suppose an n-dimensional LP-Sasakian manifold $M$ admits a quarter-symmetric non-metric $\xi$ connection, then we have the following relations on $M$ :

(i) manifold is irregular with respect to $\tilde{\nabla}$, that is, $\tilde{R}(X, Y) \xi=0$.

(ii) $\tilde{R}(X, Y) Z+\tilde{R}(Y, X) Z=0$.

(iii) $\tilde{R}(X, Y) Z+\tilde{R}(Y, Z) X+\tilde{R}(Z, X) Y=0$.

(iv) $\eta(\tilde{R}(X, Y) Z)=\eta(X)\{g(Y, Z)-g(Y, \phi Z)\}-\eta(Y)\{g(X, Z)-g(Y, \phi Z)\}$.

(v) ' $\tilde{R}(X, Y, Z, U)+{ }^{\prime} \tilde{R}(X, Y, U, Z)=\eta(Y) \eta(Z)\{g(X, U)-g(X, \phi U)\}+\eta(U) \eta(Y)\{g(X, Z)$ $-g(X, \phi Z)\}+\eta(X) \eta(Z)\{g(Y, \phi U)-g(Y, U)\}+\eta(X) \eta(U)\{g(Y, \phi Z)-g(Y, Z)\}$, where' $\tilde{R}(X, Y, Z, U)=g(\tilde{R}(X, Y) Z, U)$.

Proof. In view of the equations (2.1), (2.2) and (3.1), we have $\tilde{\nabla} \xi=0$ and hence $\tilde{R}(X, Y) \xi=0, \forall X, Y \in \chi(M)$. An $L P$-Sasakian manifold of dimension $n$ is said to be regular if $R(X, Y) \xi \neq 0$, otherwise it is irregular with respect to the Levi-Civita connection. By considering these facts, we immediately get $(i)$. Reshuffling $X$ and $Y$ in the equation (4.3) and then the sum with the equation (4.3) gives (ii). Similarly, we can prove the result (iii). In view of the equations (2.8) and (4.3), we can easily get $(i v)$. The inner product of the equation (4.3) with the vector field $U$ gives

$$
\begin{aligned}
{ }^{\prime} \tilde{R}(X, Y, Z, U)= & { }^{\prime} R(X, Y, Z, U)+g(\phi X, Z) g(\phi Y, U)+\eta(Z) \eta(Y) g(X, U) \\
& -g(X, \phi Z) \eta(Y) \eta(U)-g(\phi Y, Z) g(\phi X, U) \\
& -\eta(Z) \eta(X) g(Y, U)+g(Y, \phi Z) \eta(X) \eta(U),
\end{aligned}
$$

where ' $R(X, Y, Z, U)=g(R(X, Y) Z, U)$. Interchanging $Z$ and $U$ in the equation (4.7), we lead

$$
\begin{aligned}
{ }^{\prime} \tilde{R}(X, Y, U, Z)= & { }^{\prime} R(X, Y, U, Z)+g(\phi X, U) g(\phi Y, Z)+\eta(U) \eta(Y) g(X, Z) \\
& -g(X, \phi U) \eta(Y) \eta(Z)-g(\phi Y, U) g(\phi X, Z) \\
& -\eta(U) \eta(X) g(Y, Z)+g(Y, \phi U) \eta(X) \eta(Z) .
\end{aligned}
$$

Adding the equations (4.7) and (4.8) and then using the identity ' $R(X, Y, Z, U)+{ }^{\prime} R(X, Y, U, Z)=0$, we obtain $(v)$. Hence the statement of the Theorem 4.1 is proved.

\section{Applications to the general theory of relativity}

The goal of this section is to provide the applications of the quarter-symmetric non-metric $\xi$-connection, defined by (3.1), on an $n$-dimensional $L P$-Sasakian manifold $M$ in the general theory of relativity. Let us suppose that the $L P$-Sasakian manifold $M$ of dimension $n$ is a fluid space-time and its Ricci tensor $\tilde{S}$ with respect to $\tilde{\nabla}$ vanishes, that is, $\tilde{S}=0$. Then the equations (4.4) and (4.6) take the form

$$
S(X, Y)=(1+\psi) g(Y, \phi Z)-g(Y, Z)-n \eta(Y) \eta(Z) \text { and } r=\psi(\psi+1) .
$$

The Einstein's field equations without cosmological constant is given by

$$
S(X, Y)-\frac{r}{2} g(X, Y)=\kappa T(X, Y), \quad \forall X, Y \in \chi(M),
$$

where $T$ is the energy momentum tensor and $\kappa=8 \pi G$ (in units $c=1$ ) is the Einstein gravitational constant. If possible, we consider that the fluid space-time is a perfect fluid space-time, then the energy momentum tensor $T$ assumes the form

$$
T(X, Y)=p g(X, Y)+(p+\mu) \eta(X) \eta(Y), \quad \forall X, Y \in \chi(M),
$$

where $p$ is the isotropic pressure, $\mu$, the energy density and $\xi$ is the fluid flow velocity $(g(\xi, \xi)=-1)$ of the perfect fluid space-time. In the light of the equations (5.1) and (5.3), equation (5.2) leads to

$$
\begin{gathered}
(1+\psi) g(Y, \phi Z)-g(Y, Z)-n \eta(Y) \eta(Z)-\frac{\psi(1+\psi)}{2} g(X, Y) \\
=\kappa\{p g(X, Y)+(p+\mu) \eta(X) \eta(Y)\} .
\end{gathered}
$$

Setting $X=Y=\xi$ in the equation (5.4) and then using the equations (2.1) and (2.2), we obtain

$$
\psi(\psi+1)-2(n-1)=2 \kappa \mu .
$$


Taking a frame field and then contracting the equation (5.2), we get

$$
-(n-2) \psi(\psi+1)=2 \kappa\{p(n-1)-\mu\} .
$$

From the equations (5.5) and (5.6), we have

$$
\frac{p}{\mu}=-\frac{(n-3) \psi(1+\psi)+2(n-1)}{(n-1)\{\psi(1+\psi)-2(n-1)\}},
$$

which gives the equation of state of the perfect fluid space-time. As a particular case, we suppose that $\psi=0$ and $n=4$, then the equation of state of the perfect fluid space-times becomes

$$
3 p=\mu .
$$

G. F. R. Ellis listed in his book (see [10]) that if $\mu=3 p$, then the space-time is isotropic and homogeneous. De et al. [8] also studied the properties of the isotropic and homogeneous space-times. Thus we can state:

Theorem 5.1. Let the perfect fluid space-time admitting a quarter-symmetric non-metric $\xi$-connection $\tilde{\nabla}$ whose Ricci tensor with respect to $\tilde{\nabla}$ vanishes. If the perfect fluid space-time satisfies the Einstein's field equations without cosmological constant, then the equation of state of the space-time is $\frac{p}{\mu}=-\frac{(n-3) \psi(1+\psi)+2(n-1)}{(n-1)\{\psi(1+\psi)-2(n-1)\}}$. In particular, for $n=4$ and $\psi=0$, the space matter is filled with the isotropic and homogeneous perfect fluid.

Next we have

$$
(\tilde{R}(X, Y) \cdot \tilde{S})(Z, U)=-\tilde{S}(\tilde{R}(X, Y) Z, U)-\tilde{S}(Z, \tilde{R}(X, Y) U) .
$$

In view of the equations (2.1), (2.2), (2.7), (4.3) and the Theorem 4.1, above equation becomes

$$
\begin{aligned}
(\tilde{R}(X, & Y) \cdot \tilde{S})(Z, U)=(R(X, Y) \cdot S)(Z, U)-g(\phi X, Z) S(\phi Y, U) \\
& -\eta(Y) \eta(Z) S(X, U)+(n-1) \eta(Y) \eta(U) g(X, \phi Z) \\
& +g(\phi Y, Z) S(\phi X, U)+\eta(Z) \eta(X) S(Y, U)-(n-1) \eta(X) \eta(U) g(Y, \phi Z) \\
& -g(\phi X, U) g(\phi Y, Z)-\eta(U) \eta(Y) S(X, Z)+(n-1) \eta(Y) \eta(Z) g(X, \phi U) \\
& +g(\phi Y, U) S(\phi X, Z)+\eta(U) \eta(X) S(Y, Z)-(n-1) \eta(X) \eta(Z) g(Y, \phi U) \\
& -n\{\eta(X) \eta(U) g(Y, Z)-\eta(Y) \eta(U) g(X, Z)+\eta(Y) \eta(U) g(X, \phi Z) \\
& -\eta(X) \eta(U) g(Y, \phi Z)+\eta(X) \eta(Z) g(Y, U)-\eta(Y) \eta(Z) g(X, U) \\
& +\eta(Y) \eta(Z) g(X, \phi U)-\eta(X) \eta(Z) g(Y, \phi U)\}+\eta(Y) \eta(Z)\{g(X, \phi U) \\
& -g(X, U)\}+\eta(U) \eta(Y)\{g(X, \phi Z)-g(X, Z)\}-\eta(X) \eta(Z)\{g(Y, \phi U) \\
& -g(Y, U)\}-\eta(X) \eta(U)\{g(Y, \phi Z)-g(Y, Z)\}+(1+\psi)\left\{{ }^{\prime} R(X, Y, Z, \phi U)\right. \\
& +{ }^{\prime} R(X, Y, U, \phi Z)+g(\phi X, Z) g(\phi Y, \phi U)+\eta(Y) \eta(Z) g(X, \phi U) \\
& -g(\phi Y, Z) g(\phi X, \phi U)-\eta(X) \eta(Z) g(Y, \phi U)+g(\phi X, U) g(\phi Y, \phi Z) \\
& +\eta(Y) \eta(U) g(X, \phi Z)-g(\phi Y, U) g(\phi X, \phi Z)-\eta(U) \eta(X) g(Y, \phi Z)\},
\end{aligned}
$$

where $(R(X, Y) \cdot S)(Z, U)=-S(R(X, Y) Z, U)-S(Z, R(X, Y) U)$. If possible, we suppose that $\tilde{R} \cdot \tilde{S}=R \cdot S$, then the above equation takes the form

$$
\begin{aligned}
0= & -g(\phi X, Z) S(\phi Y, U)-\eta(Y) \eta(Z) S(X, U)+(n-1) \eta(Y) \eta(U) g(X, \phi Z) \\
& +g(\phi Y, Z) S(\phi X, U)+\eta(Z) \eta(X) S(Y, U)-(n-1) \eta(X) \eta(U) g(Y, \phi Z) \\
& -g(\phi X, U) g(\phi Y, Z)-\eta(U) \eta(Y) S(X, Z)+(n-1) \eta(Y) \eta(Z) g(X, \phi U) \\
& +g(\phi Y, U) S(\phi X, Z)+\eta(U) \eta(X) S(Y, Z)-(n-1) \eta(X) \eta(Z) g(Y, \phi U) \\
& -n\{\eta(X) \eta(U) g(Y, Z)-\eta(Y) \eta(U) g(X, Z)+\eta(Y) \eta(U) g(X, \phi Z) \\
& -\eta(X) \eta(U) g(Y, \phi Z)+\eta(X) \eta(Z) g(Y, U)-\eta(Y) \eta(Z) g(X, U) \\
& +\eta(Y) \eta(Z) g(X, \phi U)-\eta(X) \eta(Z) g(Y, \phi U)\}+\eta(Y) \eta(Z)\{g(X, \phi U) \\
& -g(X, U)\}+\eta(U) \eta(Y)\{g(X, \phi Z)-g(X, Z)\}-\eta(X) \eta(Z)\{g(Y, \phi U) \\
& -g(Y, U)\}-\eta(X) \eta(U)\{g(Y, \phi Z)-g(Y, Z)\}+(1+\psi)\left\{{ }^{\prime} R(X, Y, Z, \phi U)\right. \\
& +{ }^{\prime} R(X, Y, U, \phi Z)+g(\phi X, Z) g(\phi Y, \phi U)+\eta(Y) \eta(Z) g(X, \phi U) \\
& -g(\phi Y, Z) g(\phi X, \phi U)-\eta(X) \eta(Z) g(Y, \phi U)+g(\phi X, U) g(\phi Y, \phi Z) \\
& +\eta(Y) \eta(U) g(X, \phi Z)-g(\phi Y, U) g(\phi X, \phi Z)-\eta(U) \eta(X) g(Y, \phi Z)\} .
\end{aligned}
$$


Changing $U$ with $\xi$ in the above equation and then using the equations (2.1), (2.2) and (2.7), we obtain

$$
\eta(Y)\{S(X, Z)-(n-1) g(X, Z)\}-\eta(X)\{S(Y, Z)-(n-1) g(Y, Z)\}=0 .
$$

Again putting $X=\xi$ in (5.9), we find

$$
S(Y, Z)=(n-1) g(Y, Z) \Longrightarrow r=n(n-1),
$$

which shows that the LP-Sasakian manifold under consideration is an Einstein manifold. Thus we state:

Theorem 5.2. Let an n-dimensional Lorentzian para-Sasakian manifold $M$ equipped with a quarter-symmetric nonmetric $\xi$-connection $\tilde{\nabla}$ satisfies $\tilde{R} \cdot \tilde{S}-R \cdot S=0$, them $M$ is an Einstein space-time.

We suppose that the perfect fluid space-times satisfy $\tilde{R} \cdot \tilde{S}-R \cdot S=0$, the equations (5.2), (5.3) and (5.10) infer that

$$
2 \kappa\{p g(X, Y)+(p+\mu) \eta(X) \eta(Y)\}+(n-1)(n-2) g(X, Y)=0 .
$$

Changing $X$ and $Y$ with $\xi$ in the equation (5.11) and then using the equations (2.1) and (2.2), we have

$$
2 \kappa \mu=(n-1)(n-2),
$$

provided $n>2$. Now, considering a frame field and then contracting the equation (5.11), we lead

$$
2 \kappa\{(n-1) p-\mu\}+n(n-1)(n-2)=0 .
$$

In consequence of the equations (5.12) and (5.13), we conclude that

$$
p+\mu=0,
$$

which shows the equation of state of the perfect fluid space-time describes the Phantom Barrier. For more details, we refer [26] and the references there in. Thus we have,

Theorem 5.3. Suppose a perfect fluid space-time equipped with a quarter-symmetric non-metric $\xi$-connection $\tilde{\nabla}$ satisfies the condition $\tilde{R} \cdot \tilde{S}-R \cdot S=0$. If the perfect fluid space-time satisfies the Einstein's field equations without cosmological constant, then the model describes the evolution in the Phantom Barrier.

The conformal curvature tensor [29] plays a crucial role in the development of general theory of relativity and cosmology. It is defined as

$$
\begin{array}{r}
C(X, Y) Z=R(X, Y) Z+\frac{r}{(n-1)(n-2)}\{g(Y, Z) X-g(X, Z) Y\} \\
-\frac{1}{(n-2)}\{S(Y, Z) X-S(X, Z) Y+g(Y, Z) Q X-g(X, Z) Q Y\} .
\end{array}
$$

for all $X, Y, Z \in \chi(M)$. With the help of the equation (5.10), equation (5.15) consider the form

$$
C(X, Y) Z=R(X, Y) Z-\{g(Y, Z) X-g(X, Z) Y\} .
$$

From the above equation, it is obvious that

$$
R \cdot C=R \cdot R
$$

Eriksson and Senovilla [11], in 2010, characterized the semi-symmetric space-times and proved that these space-times are of Petrov type $\mathbf{D}, \mathbf{N}$, or $\mathbf{O}$. Hence we are in condition to state the following:

Theorem 5.4. Let a conformally semi-symmetric space-time endowed with a quarter-symmetric non-metric $\xi$-connection $\tilde{\nabla}$ satisfies $\tilde{R} \cdot \tilde{S}-R \cdot S=0$, then the space-time is of Petrov type $\mathbf{D}, \mathbf{N}$, or $\mathbf{O}$.

Next, we recall the result of Arslan et al. [1] as:

"A semi-Riemannian Einstein manifold $M, n \geq 4$, satisfies

$$
R \cdot C-C \cdot R=\frac{r}{(n-1) n} Q(g, R)=\frac{r}{(n-1) n} Q(g, C),
$$

where $Q(g, R)$ is the Tachibana tensor (see, $p-950$, Theorem 3.1, [1])."

We suppose that the space-time equipped with a quarter-symmetric non-metric $\xi$-connection satisfies $\tilde{R} \cdot \tilde{S}-$ $R \cdot S=0$ and hence the space-time is of Einstein type, that is, the equation (5.10) holds. By considering the above facts, we can write the following:

Theorem 5.5. If a space-time, $n \geq 4$, endowed with a quarter-symmetric non-metric $\xi$-connection $\tilde{\nabla}$ satisfies $\tilde{R} \cdot \tilde{S}-$ $R \cdot S=0$, then we have

$$
R \cdot C-C \cdot R=Q(g, R)=Q(g, C) .
$$




\section{Example}

Let $M=\left\{(x, y, z, u) \in \mathfrak{R}^{4}: u \neq 0\right\}$ be a 4-dimensional differentiable manifold, where $\mathfrak{R}^{4}$ denotes the set of real number of dimension 4 . Suppose that

$$
e_{1}=e^{x+u} \frac{\partial}{\partial x}, e_{2}=e^{y+u} \frac{\partial}{\partial y}, e_{3}=e^{z+u} \frac{\partial}{\partial z}, e_{4}=\frac{\partial}{\partial u}
$$

are the linearly independent vector fields and therefore it will form a basis of $M$. The Lie bracket of the vector fields $X$ and $Y$ on $M$ is defined as $[X, Y] f=X(Y f)-Y(X f)$ for any smooth function $f$. The non-vanishing components of the Lie bracket are given by

$$
\left[e_{1}, e_{4}\right]=-e_{1}, \quad\left[e_{2}, e_{4}\right]=-e_{2}, \quad\left[e_{3}, e_{4}\right]=-e_{3} .
$$

Let the semi-Riemannian metric $g$ of $M$ is defined by

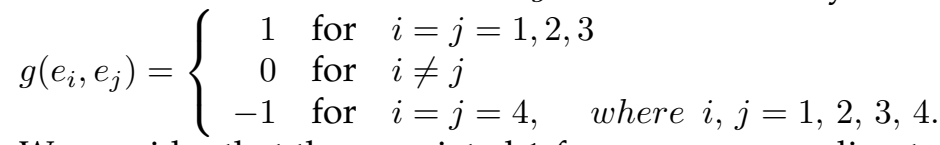

We consider that the associated 1-form $\eta$ corresponding to the unit timelike vector field $\xi=e_{4}$ of $M$ is related by the relation $g\left(X, e_{4}\right)=\eta(X), \forall X \in \chi(M)$. Let the $(1,1)$-tensor field $\phi$ is defined by

$$
\phi e_{1}=-e_{1}, \quad \phi e_{2}=-e_{2}, \quad \phi e_{3}=-e_{3}, \quad \phi e_{4}=0,
$$

then it is obvious that

$$
\phi^{2} X=X+\eta(X) e_{4}, \quad \eta\left(e_{4}\right)=-1
$$

hold for all $X \in \chi(M)$. Thus the manifold $M$ equipped with the compatible metric $g$ is a 4-dimensional Lorentzian para-contact metric manifold. With the help of the Koszul's formula,

$$
\begin{aligned}
2 g\left(\nabla_{X} Y, Z\right) & =X g(Y, Z)+Y g(Z, X)-Z g(X, Y) \\
& -g(X,[Y, Z])-g(Y,[X, Z])+g(Z,[X, Y]),
\end{aligned}
$$

we can easily find that

$$
\begin{aligned}
& \nabla_{e_{i}} e_{4}=-e_{i}, \quad i,=1,2,3 \\
& \nabla_{e_{i}} e_{i}=-2 e_{4}, \quad i,=1,2,3 \\
& \nabla_{e_{i}} e_{j}=0, \quad i \neq j, \text { and } i=j=4 .
\end{aligned}
$$

From the above discussions, we found that $\nabla_{X} e_{4}=\phi X, \forall X \in \chi(M)$ and hence we can say that $(M, g)$ is a Lorentzian para-Sasakian manifold of dimension 4 . The non-vanishing components of the curvature tensor $R$ of the Levi-Civita connection $\nabla$ are

$$
\begin{array}{llll}
R\left(e_{1}, e_{2}\right) e_{1}=-e_{2}, & R\left(e_{1}, e_{3}\right) e_{1}=-e_{3}, & R\left(e_{1}, e_{4}\right) e_{1}=-e_{4}, & R\left(e_{1}, e_{2}\right) e_{2}=e_{1}, \\
R\left(e_{2}, e_{3}\right) e_{2}=-e_{3}, & R\left(e_{2}, e_{4}\right) e_{2}=-e_{4}, & R\left(e_{1}, e_{3}\right) e_{3}=e_{1}, & R\left(e_{2}, e_{3}\right) e_{3}=e_{2}, \\
R\left(e_{3}, e_{4}\right) e_{3}=-e_{4}, & R\left(e_{1}, e_{4}\right) e_{4}=-e_{1}, & R\left(e_{2}, e_{4}\right) e_{4}=-e_{2}, & R\left(e_{3}, e_{4}\right) e_{4}=-e_{2} .
\end{array}
$$

Also, the non-vanishing components of the Ricci tensor $S$ are listed as

$$
S\left(e_{4}, e_{4}\right)=-3, \quad S\left(e_{i}, e_{i}\right)=3, i=1,2,3 .
$$

It is obvious that $R \cdot S=0$ and $S=(n-1) g$ for $n=4$.

Next, with the help of the above discussions, the linear connection $\tilde{\nabla}$ defined on $M$ by the equation (3.1) takes the form

$$
\tilde{\nabla}_{e_{i}} e_{i}=-2 e_{4} \text { for } i=1,2,3 \text { and } \tilde{\nabla}_{e_{i}} e_{j}=0 \text { otherwise. }
$$

The torsion tensor $\tilde{T}$ of $\tilde{\nabla}$ defined by $\tilde{T}(X, Y)=\tilde{\nabla}_{X} Y-\tilde{\nabla}_{Y} X-[X, Y]$ for all $X$ and $Y$ on $M$. Let $X$ and $Y$ are the arbitrary vector fields of $M$, then we have $X=X^{1} e_{1}+X^{2} e_{2}+X^{3} e_{3}+X^{4} e_{4}$ and $Y=Y^{1} e_{1}+Y^{2} e_{2}+Y^{3} e_{3}+$ $Y^{4} e_{4}$. Thus

$$
\tilde{T}(X, Y)=\left(Y^{4} X^{1}-X^{4} Y^{1}\right) e_{1}+\left(Y^{4} X^{2}-X^{4} Y^{2}\right) e_{2}+\left(Y^{4} X^{3}-X^{4} Y^{3}\right) e_{3} .
$$


Again,

$$
g\left(Y, e_{4}\right) \phi X-g\left(X, e_{4}\right) \phi Y=\left(Y^{4} X^{1}-X^{4} Y^{1}\right) e_{1}+\left(Y^{4} X^{2}-X^{4} Y^{2}\right) e_{2}+\left(Y^{4} X^{3}-X^{4} Y^{3}\right) e_{3} .
$$

This shows that the equation (1.1) is satisfied and hence the linear connection $\tilde{\nabla}$ is a quarter-symmetric connection on $M$. The straight forward calculation shows that

$$
\left(\tilde{\nabla}_{e_{i}} g\right)\left(e_{i}, e_{4}\right)=-2 \neq 0, \quad i=1,2,3 .
$$

This reflects that the quarter-symmetric connection $\tilde{\nabla}$ is non-metric. Also we have $\tilde{\nabla}_{e_{i}} e_{4}=0, i=1,2,3,4$. Thus the connection $\tilde{\nabla}$ defined on $M$ by the equation (3.1) is a quarter-symmetric non-metric $\xi$-connection. The nonzero components of the curvature tensor $\tilde{R}$ with respect to the quarter-symmetric non-metric $\xi$-connection $\tilde{\nabla}$ are

$$
\begin{aligned}
& \tilde{R}\left(e_{1}, e_{4}\right) e_{1}=\tilde{R}\left(e_{2}, e_{4}\right) e_{2}=\tilde{R}\left(e_{3}, e_{4}\right) e_{3}=-2 e_{4}, \\
& \tilde{S}\left(e_{1}, e_{1}\right)=\tilde{S}\left(e_{2}, e_{2}\right)=\tilde{S}\left(e_{3}, e_{3}\right)=2,
\end{aligned}
$$

which follows that $\tilde{R} \cdot \tilde{S}=0$. Thus we conclude that $\tilde{R} \cdot \tilde{S}-R \cdot S=0$ and $S=(n-1) g$. Hence the manifold under consideration is an Einstein manifold. Therefore the Theorem 5.2 is verified.

\section{Acknowledgments}

The authors express their sincere thanks to the referees and the Editor for proving the valuable suggestions in the improvement of the paper.

\section{References}

[1] Arslan, K., Deszcz, R., Ezentas, R., Hotloś, M. and Murathan, C., On generalized Robertson-Walker spacetimes satisfying some curvature condition. Turk J Math 38 (2014), 353-373.

[2] Aqeel, A. A., De, U. C. and Ghosh, G. C., On Lorentzian para-Sasakian manifolds. Kuwait J. Sci. Eng. 31 (2004), no. 2, 1-13.

[3] Barman, A., Weakly symmetric and weakly Ricci-symmetric $L P$-Sasakian manifolds admitting a quarter-symmetric metric connection. Novi Sad J. Math. 45 (2015), no. 2, 143-153.

[4] Cartan, E., Sur une classe remarquable d'espaces de Riemannian. Bull. Soc. Math. France 54 (1926), 214-264.

[5] Chaubey, S. K. and Ojha, R. H., On semi-symmetric non-metric and quarter-symmetric metric connections. Tensor N. S. 70 (2008), no. 2, 202-213.

[6] Chaubey, S. K., Lee, J. W. and Yadav, S., Riemannian manifolds with a semi-symmetric metric P-connection. J. Korean Math. Soc. 56 (2019), no. $4,1113-1129$.

[7] De, U. C., Matsumoto, K. and Shaikh, A. A., On Lorentzian para-Sasakian manifolds. Rendiconti del Seminario Mat. de Messina 3 (1999), 149-156.

[8] De, U. C., Velimirović, L. and Mallick, S., On a type of spacetime. International Journal of Geometric Methods in Modern Physics 14 (2017), no. 1,1750003 (9 pages).

[9] Demirba ğ, S. A., Yilmaz, H. B., Uysal, S. A. and Zengin, F. Ö., On quasi Einstein manifolds admitting a Ricci quarter-symmetric metric connection. Bull. of Math. Anal. and Appl. 3 (2011), no. 4, 84-91.

[10] Ellis, G. F. R., Relativistic Cosmology in 'General Relativity and Cosmology', ed. R. K. Sachs, Academic Press, London, 1971.

[11] Eriksson, I. and Senovilla, J. M. M., Note on (conformally) semi-symmetric spacetimes. Class. Quantum Grav. 27 (2010), 027001.

[12] Friedmann, A. and Schouten, J. A., Uber die Geometry der halbsymmetrischen Ubertragung. Math Zeitschr 21 (1924), $211-223$.

[13] Golab, S., On semi-symmetric and quarter-symmetric linear connections. Tensor N. S. 29 (1975), 249-254.

[14] Güler, S. and Demirba $\breve{g}$, S. A., A study of generalized quasi Einstein spacetimes with applications in general relativity. Int J Theor Phys 55 (2016), 548-562.

[15] Hayden, H. A., Subspace of space with torsion. Proc. London Math. Soc. 34 (1932), 27-50.

[16] Mantica, C. A., De, U. C., Suh, Y. J. and Molinari, L. G., Perfect fluid spacetimes with harmonic generalized curvature tensor. Osaka J. Math. 56 (2019), 173-182.

[17] Matsumoto, K., On Lorentzian para-contact manifolds. Bull. Yamagata Univ. Nat. Sci. 12 (1989), 151-156.

[18] Matsumoto, K. and Mihai, I., On a certain transformation in a Lorentzian para-Sasakian manifold. Tensor N. S. 47 (1988), 189-197.

[19] Mihai, I. and Rosca, R., On Lorentzian P-Sasakian manifolds. Classical Analysis, World Scientific Publi., Singapore (1992), 155-169.

[20] Mihai, I., De, U. C. and Shaikh, A. A., On Lorentzian para-Sasakian manifolds. Korean J. Math. Sci. 6 (1999), 1-13.

[21] Mishra, R. S. and Pandey, S. N., On quarter symmetric metric F-connections. Tensor N. S. 34 (1980), 1-7.

[22] Murathan, C., Yildiz, A., Arslan, K. and De, U. C., On a class of Lorentzian para-Sasakian manifolds. Proc. Estonian Acad. Sci. Phys. Math. 55 (2006), no. 4, 210-219.

[23] Rastogi, S. C., On quarter-symmetric metric connection. C. R. Acad. Bulg. Sci. 31 (1978), no. 8, 811-814.

[24] Patterson, E. M., Some theorems on Ricci-recurrent spaces. J. London Math. Soc. 27 (1952), 287-295.

[25] Prasad, R. and Haseeb, A., On a Lorentzian para-Sasakian manifold with respect to the quarter-symmetric metric connection. Novi Sad J. Math. 46 (2016), no. 2, 103-116. 
[26] Srivastava, S. K., Scale factor dependent equation of state for curvature inspired dark energy, phantom barrier and late cosmic acceleration. Physics Letters B 643 (2006), 1-4.

[27] Sular, S., Özgür, C. and De, U. C., Quarter-symmetric metric connection in a Kenmotsu manifold. SUT Journal of Mathematics 44 (2008), 297-306.

[28] Szabó, Z. I., Structure theorems on Riemannian spaces satisfying $R(X, Y) \cdot R=0$. I. The local version. J. Diff. Geom 17 (1982), $531-582$.

[29] Weyl, H., Reine Infinitesimalgeometrie. Math. Z. 2 (1918), 384-411.

[30] Zengin, F. Ö., M-projectively flat spacetimes. Math. Rep. 4 (2012), no. 4, 363-370.

\section{Affiliations}

\section{SUDHAKAR K CHAUBEY}

AdDRESS: Section of Mathematics, Department of Information Technology, Shinas College of Technology, Shinas, P.O. Box 77, Postal Code 324, Oman.

E-MAIL: sk22_math@yahoo.co.in; sudhakar.chaubey@shct.edu.om

\section{UDAY CHAND DE}

ADDRESS: Department of Pure Mathematics, University of Calcutta 35, Ballygaunge Circular Road Kolkata 700019, West Bengal, India.

E-MAIL: uc_de@yahoo.com 